\title{
Negociaciones intergubernamentales en la Unión Europea: estrategias y poder*
}

\author{
Intergovernmental Negotiations in the European Union: \\ Strategies and Power
}

\author{
Gemma Mateo González
}

Universidad de Salzburgo

doi: 10.18543/ced-55-2016pp85-118

Sumario: I. Introducción.-II. El funcionamiento del Consejo y del Consejo Europeo de la UE.-III. Estrategias. 1. La sombra del futuro. 2. Preferencias y la política domestica. 3. El poder de los Estados. 4. Instituciones. 5. Cultura. 6. Estadio o nivel de las negociaciones. 7. Temas. 8. Euroescepticismo.-IV. Poder. 1. Poder del voto. 2. Poder institucional. 3. Poder estructural. 4. Características de los negociadores. 5. El BATNA de los países. 6. Las coaliciones entre países.-V. Conclusiones.

\begin{abstract}
Resumen: En el marco del Consejo de la Unión Europea (UE) se llevan a cabo más de 6.000 reuniones anuales. Muchas de éstas tienen como objetivo preparar reuniones a más alto nivel: entre Ministros y entre los Jefes de Estado y/o de Gobierno. La relevancia y trascendencia de estas reuniones es indiscutible de ahí que exista una creciente literatura que analiza distintos aspectos de estas negociaciones. El objetivo de este artículo es hacer una revisión de esta literatura a partir de dos cuestiones: La primera se refiere a las estrategias que utilizan los Estados en las negociaciones. En definitiva, ¿cómo se negocia en la UE? ¿Qué Estados optan por estrategias cooperativas y cuáles por estrategias conflictivas? Basándonos en la literatura derivamos una serie de factores que pueden determinar la elección de estrategias: La sombra del futuro, las preferencias domésticas, el poder, la cultura, el nivel o estadio de la negociación, el tema y el euroescepticismo. La segunda pregunta consiste en analizar qué Estados tienen más poder en las negociaciones. Analizamos algunos factores que pueden explicar la distribución de poder: el poder del voto, el poder institucional, el poder estructural, las características de los negociadores, el BATNA de los países y las coaliciones. A partir de esta revisión de literatura, el artículo ofrece algunas conclusiones y sugerencias para futuras investigaciones.
\end{abstract}

Palabras clave: negociaciones, Consejo, Consejo Europeo, estrategias, poder.

* Recibido el 19 de mayo de 2016, aceptado el 28 de junio de 2016. 
Abstract: More than six thousand meetings take place each year in the framework of the Council of the European Union (EU). The objective of many of these meetings is to prepare meetings at the highest level: between Ministers and Heads of State and/or Government. As the relevance and importance of these meetings is undisputed, there is a growing literature that analyzes different aspects of these negotiations. The aim of this article is to review this literature starting from two major questions: The first refers to the strategies used by different Member States in the negotiations. In short, how do states negotiate in the EU? Which states opt for cooperative strategies and which ones for conflictive strategies? Building on the literature, the article derives a number of factors that can determine the choice of strategies: the shadow of the future, domestic preferences, power, culture, level or stage of the negotiation, the subject area and Euroscepticism. The second question is to analyse which states have most power in these negotiations. Various factors appear in different studies to explain the distribution of power: voting power, institutional power, structural power, the characteristics of the negotiators, the BATNA of countries and coalitions between countries. Building on this review of the literature, the article presents some conclusions and some ideas for future research.

Keywords: negotiations, Council, European Council, strategies, power.

\section{Introducción}

En la Unión Europea (UE) se celebran alrededor de 6.000 reuniones anuales a muy distintos niveles en el Consejo y en el Consejo Europeo. ${ }^{1}$ Muchas de estas reuniones tienen como objetivo preparar posteriores encuentros a más alto nivel ya sea entre los Ministros o entre los Jefes de Estado y/o de Gobierno en las grandes cumbres. Son las instituciones más poderosas en el proceso de toma de decisiones de la UE. ${ }^{2}$ La relevancia y trascendencia de estas instituciones es indiscutible de ahí que exista una creciente literatura que analiza distintos aspectos de estas negociaciones. El objetivo de este artículo es hacer una revisión de estos estudios explorando lo que hemos aprendido sobre las estrategias y el poder en las negociaciones intergubernamentales en la UE, es decir en el Consejo y en el Consejo

1 Jeffrey Lewis, «The Council of the European Union and the European Council», en Politics in the European Union, Fourth Edition, ed. por Michelle Cini y Nieves Perez-Solorzano Borragan (Oxford: Oxford University Press, 2013), 220. Después del Tratado de Lisboa (2009), el Consejo de la Unión Europea ha pasado a denominarse simplemente Consejo y el Consejo Europeo pasa a ser formalmente una nueva institución.

2 Entre muchos otros: Martin Westlake y David Galloway, eds., The Council of the European Union. (London: John Harper Publishing, 2006); Fiona Hayes-Renshaw y Helen Wallace The Council of Ministers (London: Macmillan, 1997). 
Europeo. El carácter intergubernamental de este tipo de negociaciones hace idóneo su estudio por distintos motivos. ${ }^{3}$ Principalmente porque se trata de negociaciones entre los gobiernos de los Estados miembros, aunque esté presente la Secretaria General y la Comisión Europea, el poder de voto recae únicamente en los países. Además, existen otras organizaciones internacionales que tienen órganos similares de ahí que permita exportar su estudio a otras organizaciones y, por tanto, llevar a cabo comparaciones interesantes. Por último, revisar la literatura es un ejercicio de gran utilidad para apreciar tanto los logros alcanzados como las carencias en este campo de investigación.

El secretismo que rodea estas negociaciones dificulta su estudio. Las reuniones en el Consejo Europeo se llevan a cabo a puerta cerrada y surgen de ellas escasos documentos públicos, prácticamente sólo se conocen los resultados. Existe muy poca información sobre lo que acontece en la sala con la excepción de algunas anécdotas que reproduce la prensa. De ahí que la mayoría de investigadores recurran a las entrevistas personales con los negociadores para saber más sobre el fondo de las negociaciones. Aunque las entrevistas son una fuente primaria de indudable valor, siempre hay incerteza sobre la veracidad de la información y, sobre todo, su objetividad. La situación es similar en el Consejo cuyas reuniones siguen siendo en su mayoría restringidas a pesar de algunos intentos de hacerlas más transparentes. Tampoco se sabe mucho de los cientos de comités que se reúnen regularmente para discutir distintos temas.

A pesar de esta importante restricción, en los últimos años se han incrementado el número de estudios sobre distintos aspectos de estas negociaciones. Algunos trabajos han tratado de aproximarse a preguntas claves como, por ejemplo, ¿Cómo negocian los Estados? ¿Qué estrategias utilizan? ¿Quiénes tienen más poder y por qué? ¿Qué factores determinan el poder entre los Estados? Precisamente, el objetivo de este artículo es resumir el avance de estos estudios y ver hasta qué punto se ha dado respuesta a algunas de estas cuestiones.

El artículo está dividido en cinco partes: después de la introducción, viene una sección dedicada al funcionamiento del Consejo y del Consejo Europeo. Describimos brevemente cómo se llevan las negociaciones en cada uno de los niveles - desde el más técnico a nivel de comités hasta el

${ }^{3}$ Sobre el funcionamiento de estas instituciones véase entre otros: Fiona Hayes-Renshaw y Helen Wallace, The Council of Ministers, (New York, Palgrave Macmillan: 2006); Philippa Scherrington The Council of Ministers: Political Authority in the European Union (London: Pinter, 2000); Jonas Tallberg, Bargaining Power in the European Council. (Working Paper SIEPS 1, 2007); Daniel Naurin y Helen Wallace, eds., Unveiling the Council of the European Union: Games governments play in Brussels (Basingstoke: Palgrave Macmillan, 2008). 
Consejo Europeo - mencionando los cambios introducidos en el Tratado de Lisboa (2009). En la siguiente sección analizamos la literatura sobre estrategias y distinguimos una serie de factores que determinan la elección de las estrategias de los países. A continuación, estudiamos una serie de factores que pueden explicar la distribución del poder entre los Estados. Por último, en las conclusiones ofrecemos algunas pistas sobre cómo proseguir en la investigación.

\section{El funcionamiento del Consejo y del Consejo Europeo de la UE}

En los últimos años la labor que desempeña, tanto el Consejo como el Consejo Europeo, se ha ido haciendo cada vez más compleja por el aumento de Estados miembros y por el creciente número de temas en discusión. En los años cincuenta eran seis los países sentados en la mesa de negociaciones, en la actualidad están 28 países representados. El avance en el proceso de integración europea ha supuesto también un aumento de legislación que debe ser aprobada en las distintas formaciones del Consejo. Esta situación ha hecho necesario llevar a cabo una serie de cambios en el funcionamiento de ambas instituciones con el fin de hacer las negociaciones más eficientes.

El Consejo y el Consejo Europeo son las dos instituciones claves en el proceso de toma de decisiones de la UE. Los Estados miembros están siempre representados en los distintos niveles - desde los grupos de trabajo del Consejo hasta las grandes cumbres entre los Jefes de Estado y/o de Gobierno- - La Presidencia dirige las reuniones asistida por la Secretaria General y la Comisión Europea está presente en todas las reuniones. Se celebran tres o cuatro Consejos Europeos al año, aunque en los últimos años ha habido un incremento como consecuencia de los problemas de la crisis económica en la zona Euro. ${ }^{4}$ Las reuniones entre las distintas formaciones ministeriales oscilan entre dos o cuatro por año, dependiendo de la formación y de la legislación pendiente de aprobar. Algunos comités o grupos de trabajo pueden llegar a reunirse varias veces a la semana. ${ }^{5}$

El Consejo es el encargado de aprobar nueva legislación y reúne principalmente a todos los Ministros de los Estados miembros, en su ausencia pueden ocupar su silla los Secretarios de Estado. Está representado en diez formaciones distintas: agricultura y pesca; asuntos económicos y financieros; asuntos exteriores; asuntos generales; competitividad; educación, ju-

\footnotetext{
${ }^{4}$ Lewis, «The Council of the European Union and the European Council», 221.
}

5 Lewis, «The Council of the European Union and the European Council», 220. 
ventud, cultura y deporte; empleo, política social, sanidad y consumidores; justicia y asuntos de interior; medio ambiente; transporte, telecomunicaciones y energía. Decide por mayoría cualificada o por consenso. Aunque desde el 2014 el sistema habitual de votación del Consejo es la mayoría cualificada en el $80 \%$ de la legislación, los Ministros recurren a menudo al consenso. ${ }^{6}$ En la actualidad, la Presidencia la ocupan tres países por un plazo de año y medio. Para mejorar la eficiencia y continuidad de su trabajo, se crea un programa de trabajo conjunto que ocupa tres presidencias. ${ }^{7}$ La única excepción afecta al Consejo de Asuntos Exteriores que está presidido por el nuevo Representante para Asuntos Exteriores y Política de Seguridad. La Secretaria General asiste a la Presidencia y da asesoramiento jurídico además de ofrecer ayuda administrativa. Para simplificar el trabajo del Consejo, la agenda de trabajo está siempre dividida en puntos A y B: los puntos A se aprueban sin debate ya que han sido previamente aprobados en el COREPER (Comité de Representantes Permanentes de la UE), mientras que los puntos B son los temas sobre los que los Ministros tienen que discutir.

Las reuniones de los Ministros vienen precedidas de las reuniones del COREPER que es el órgano encargado de preparar y coordinar la agenda de las reuniones entre Ministros. ${ }^{8}$ Tiene dos formaciones: COREPER I y COREPER II. El primero está compuesto por los representantes permanentes adjuntos de cada país y tiene como principal misión preparar el trabajo de las formaciones del Consejo. Asimismo, sus reuniones son preparadas por el grupo «Mertens» el cual adelanta las posiciones de las delegaciones ante el COREPER I. El COREPER II está compuesto por los embajadores en la UE y se ocupa de aspectos relativos a las formaciones de asuntos generales, asuntos exteriores, asuntos económicos y financieros, justicia y asuntos de interior del Consejo. Su trabajo es también preparado por un grupo informal, el grupo de los «Antici,» cuya función, a partir de las posiciones de los países, es organizar las reuniones del COREPER II. Las dos configuraciones del COREPER se reúnen todas las semanas así como los grupos de seguimiento.

Además del COREPER, el Consejo en general está asistido por más de 150 grupos de trabajo y comités especializados en muy distintos ámbi-

${ }^{6}$ El voto por mayoría cualificada en el Consejo a partir de noviembre de 2014 requiere: un mínimo del 55\% de los miembros del Consejo, con un mínimo de 15 miembros, y el 65\% de la población.

7 Jonas Tallberg Leadership and Negotiation in the European Union: The Power of the Presidency. (Cambridge: Cambridge University Press, 2006).

8 Véase sobre el COREPER: Jeffrey Lewis «Is the "Hard Bargaining" Image of the Council Misleading? The Committee of Permanent Representatives and the Local Elections Directive». Journal of Common Market Studies, 36, n. ${ }^{\circ}$ (1998): 479-504. 
tos. Estos grupos se dividen en dos categorías según el órgano que los ha creado. Por un lado, están los comités creados por los tratados, por decisión intergubernamental o por mandato del Consejo para un tema específico. Por otro lado, están los comités que crea el mismo COREPER y que se ocupan de temas concretos según las nuevas demandas. La gran diferencia entre ambos es que los primeros suelen ser permanentes mientras que los segundos tienen un carácter temporal. Existen también los comités que se denominan ad hoc y que responden a demandas concretas cuyo objetivo y mandato es muy específico. El número de comités es muy elevado y varía en función de la actividad del Consejo. Como ejemplo, en el 2011 hubo un total de 4.373 reuniones de los comités, es decir, algo más de 80 reuniones por semana. ${ }^{9}$

El Consejo Europeo es la institución más poderosa en la UE. ${ }^{10}$ Aprueba por unanimidad de todos sus miembros las decisiones más trascendentales sobre el proceso de integración europea. Está compuesto por los Jefes de Estado o de Gobierno de los Estados miembros acompañados generalmente por el Ministro de Asuntos Exteriores quien puede ceder el puesto a su homólogo de economía cuando se discuten temas económicos. El Tratado de Lisboa (2009) ha introducido la figura del nuevo presidente del Consejo Europeo cuya función es preparar y coordinar los trabajos de la institución. El nuevo presidente no representa a su país y su mandato es de dos años y medio. Por definición, tiene que ser imparcial y su objetivo es hacer progresar las negociaciones. ${ }^{11}$ Este cambio pretende dar una mayor estabilidad y continuidad a la institución. La elección y restitución del presidente, si fuese el caso, debe ser aprobada por mayoría cualificada entre sus miembros.

La Secretaria General asiste al Presidente y la Comisión Europea puede estar presente aunque no tiene poder de voto. Las reuniones son secretas y el acceso a la sala es absolutamente restringido. Sólo puede acceder a la sala el Antici de cada país para recoger o entregar una nota, facilitar un documento o atender a alguna petición concreta. El Consejo Europeo se rige por una serie de reglas informales que, aunque no están escritas, se siguen de modo preciso. ${ }^{12}$ La Secretaria General se encarga de tomar notas de todo lo que acontece en la sala. Estas notas las reciben los Antici (uno por delegación) los cuales a su vez tienen que transmitir la información de lo que sucede a sus delegaciones con la mayor rapidez posible. Los documentos fi-

${ }^{9}$ Lewis, «The Council of the European Union and the European Council», 220.

${ }_{10}$ Westlake y Galloway. Eds, The Council...; Jan Werts, The European Council (London: John Harper Publishing, 2008).

11 Tallberg Leadership....

12 Mateo, Gemma. Hacia una Constitución Europea: Las Conferencias Intergubernamentales en la Unión Europea (Valencia: Tirant Lo Blanch, 2008). 
nales de los Consejos Europeos son las conclusiones que ofrecen un breve resumen de los logros alcanzados. A menudo sugieren nuevos temas en los que se debería seguir discutiendo. Las decisiones en el Consejo Europeo se toman por unanimidad, lo que significa que cualquier Estado miembro tiene la capacidad de vetar una decisión. Esta situación presupone teóricamente que todos los países tienen el mismo poder en las negociaciones.

\section{Estrategias}

Por estrategias nos referimos a «una serie de comportamientos» y por tácticas a «las acciones particulares que constituyen o forman parte de una estrategia». ${ }^{13}$ Existen distintas clasificaciones, entre ellas: Lax y Sebenius distinguen entre «value-claiming» versus «value-creating» ${ }^{14}$; Walton y McKersie se refieren a «distributive» $\mathrm{y}$ «integrative bargaining» ${ }^{15}$; Hopmann distingue entre «bargaining» $\mathrm{y}$ «problem solving» ${ }^{16}$; Niemann entre «strategic action» $\mathrm{y}$ «communicative action» ${ }^{17}$; Risse entre «bargaining» $\mathrm{y}$ «arguing» ${ }^{18}$ y Dür y Mateo y Hopmann entre «hard» y «soft bargaining». ${ }^{19}$ El problema de estas clasificaciones es que se refieren a tipos ideales mientras que se espera que en el mundo real los negociadores combinen diferentes tácticas. Además, estas tipologías se basan en la intencionalidad de los actores, presuponiendo que actúan en defensa del interés común o bien en defensa de sus propios intereses, cuando lo más probable es que independientemente del tipo de tácticas que escojan su objetivo será siempre el mismo: aumentar la porción del pastel que reciben. Otra dificultad es iden-

${ }^{13}$ Odell, John. Negotiating the World Economy (New York: Cornell University Press, 2000), 31 .

${ }^{14}$ Lax, David A. y James Sebenius. The Manager as Negotiator: Bargaining for Cooperation and Competitive Gain (New York: Free Press, 1986).

15 Walton, Richard E. y Robert B. McKersie, A Behavioral Theory of Labor Negotiations: An Analysis of a Social Interaction System (Ithaca: ILR Press, 1965).

${ }^{16}$ Hopmann, Terrence P. «Two Paradigms of Negotiation: Bargaining and Problem Solving» Annals of the American Academy of Political and Social Science 542 (1995): 24-47.

17 Niemann, Arne, «Between Communicative Action and Strategic Action: The Article 113 Committee and the Negotiations on the WTO Basic Telecommunications Services Agreement» Journal of European Public Policy 11, n. 3 (2004): 379-407.

18 Risse, Thomas «Let's Argue: Communicative Action in World Politics» International Organization 54, n. ${ }^{\circ} 1$ (2000): 1-40.

19 Dür, Andreas y Gemma Mateo, «Actor Characteristics and Negotiation Tactics in the EU» Journal of European Public Policy 17, n. 5 (2010a): 680-93; Dür, Andreas y Gemma Mateo, «Bargaining Power and Negotiation Tactics: The Negotiations on the EU's Financial Perspective, 2007-2013» Journal of Common Market Studies 48, n. ${ }^{\circ} 3$ (2010b): 557-78; Terrence P. Hopmann «Bargaining in Arms Control Negotiations: The Seabeds Denuclearization Treaty» International Organization 28, n. $^{\circ} 3$ (1974): 313-43. 
tificar que tácticas hacen parte de cada grupo, es decir, si hacen parte de la dimensión cooperativa o, por el contrario, conflictiva.

Basándose en el trabajo inicial de Hopmann, ${ }^{20}$ Dür y Mateo definen «soft bargaining» como el uso de tácticas cooperativas o amistosas mientras que «hard bargaining» se caracteriza por la utilización de tácticas más conflictivas o agresivas. ${ }^{21}$ A partir de la definición de Odell, ${ }^{22}$ Dür y Mateo derivan una lista de tácticas para ambos tipos de comportamiento. ${ }^{23}$ Siguiendo esta distinción, el primer tipo incluye: «indicar flexibilidad, hacer una declaración conciliadora, elogiar a la otra parte, buscar aliados para alcanzar un compromiso, hacer una propuesta de compromiso;» mientras que en el segundo tipo de estrategias están: «comprometerse a no ceder, criticar a la otra parte, instigar o hacer parte de una coalición defensiva y hacer amenazas». Esta clasificación además de ser novedosa permite examinar a nivel empírico de modo sistemático el comportamiento de los actores.

Asimismo, McKibben ofrece otra clasificación de estrategias basada en cuatro tipos que distingue en función del grado de confrontación. ${ }^{24}$ En los dos extremos están las estrategias «cooperativas» y las estrategias «confrontativas». Las primeras se caracterizan porque los negociadores dan muestras de ofrecer concesiones mientras que las segundas se caracterizan por las amenazas. Entre medio de estos dos extremos, McKibben se refiere a las estrategias que denomina «algo cooperativas» y «algo confrontativas». ${ }^{25}$

A continuación, vamos a examinar una serie de factores o variables que, teóricamente, pueden influir en la elección de las estrategias de los negociadores en el Consejo y en el Consejo Europeo.

${ }^{20}$ Hopmann, «Bargaining in Arms Control Negotiations: The Seabeds Denuclearization Treaty», 318 .

${ }^{21}$ Dür y Mateo, «Bargaining Power and Negotiation Tactics: The Negotiations on the EU's Financial Perspective, 2007-2013».

22 Odell, Negotiating...

${ }^{23}$ Dür y Mateo, «Bargaining Power and Negotiation Tactics: The Negotiations on the EU's Financial Perspective, 2007-2013», 563. También han elaborado similares listas de tácticas en negociaciones: Walton, Richard y Robert McKersie, A Behavioral Theory of Labor Negotiations: An Analysis of a Social Interaction System. (Ithaca, New York: ILR Press, 1965). Elms, Deborah, «How bargaining alters outcomes: bilateral trade negotiations and bargaining strategies» International Negotiation, 11, n. 3 (2006): 399-429. John Odell, Negotiating the World Economy. (New York: Cornell University Press, 2000).

${ }^{24}$ Heather McKibben, State strategies in international bargaining: Play by the rules or change them? (Cambridge: Cambridge University Press, 2015), 116.

${ }_{25}$ McKibben, State..., 117. 


\section{La sombra del futuro}

El argumento más establecido sobre los determinantes de las estrategias de negociación entre los Estados miembros en la UE es que la permanente celebración de reuniones produce «un modo de negociación cooperativo». ${ }^{26}$ En los años ochenta, Keohane ya llego a referirse a esta situación como «la sombra del futuro.» ${ }^{27} \mathrm{La}$ idea principal es que sabiendo que van a reunirse repetidamente, los negociadores desarrollan un estilo de negociación particular. ${ }^{28}$ Según Héritier, los negociadores son conscientes de que las negociaciones no son temporales sino duraderas. ${ }^{29}$ Lewis denomina este modo de tomar decisiones como el método comunitario. ${ }^{30}$ Este se caracteriza por una cierta reciprocidad, una profunda confianza, una mutua responsabilidad, una búsqueda de consenso y una cultura del compromiso.

En particular, este argumento se ha extendido en los estudios enfocados en los niveles más inferiores o técnicos del Consejo, es decir, en el COREPER donde la interacción es continua. Lewis, en concreto, asegura que el COREPER es «un importante mecanismo de socialización del método comunitario.» ${ }^{31}$ Los miembros se identifican como parte de un grupo, tienen una visión muy parecida sobre los temas que discuten y hacen parte de un sistema en que predomina «la cultura del compromiso». ${ }^{32}$ Lewis destaca que «el entorno institucional del Consejo ha inculcado preferencias co-

${ }^{26}$ Elgström, Ole y Christer Jönsson, «Negotiating in the European Union: bargaining or problem solving» Journal of European Public Policy 7, n. 5 (2000): 687.

27 Robert O. Keohane, After Hegemony: Cooperation and Discord in the World Political Economy (Princeton: Princeton University Press, 1984)

28 Véase entre otros: Bailer, Stephanie, «What Factors Determine Bargaining Power in the EU?» Journal of European Public Policy 17, n. 5 (2010): 743-52.; Elgström y Jönsson, «Negotiating in the European Union: bargaining or problem solving»; Lewis «Is the "Hard Bargaining" Image of the Council Misleading? The Committee of Permanent Representatives and the Local Elections Directive»; Thomas, Daniel, «Explaining the negotiation of EU foreign policy: Normative institutionalism and alternative approaches» International Politics, 46 (2009): 339-57;

${ }^{29}$ Héritier, Adrienne, «The accommodation of diversity in European policymaking and its outcomes: regulatory policy as a patchwork» Journal of European Public Policy 3, n. ${ }^{\circ} 2$ (1996): 149-67.

${ }^{30}$ Lewis, Jeffrey «The Methods of Community in EU Decision-making and Administrative Rivalry in the Council's Infrastructure» Journal of European Public Policy, 7, n. ${ }^{\circ} 2$ (2000): 261-89.

${ }^{31}$ Lewis «Is the "Hard Bargaining" Image of the Council Misleading? The Committee of Permanent Representatives and the Local Elections Directive», 486.

${ }^{32}$ Lewis «Is the "Hard Bargaining" Image of the Council Misleading? The Committee of Permanent Representatives and the Local Elections Directive», 498. 
lectivas intrínsecas para la negociación cooperativa». ${ }^{33} \mathrm{El}$ autor se refiere a cuatro variables que impactan en el comportamiento de los actores: el grado de aislamiento, el alcance y la amplitud de la interacción, la intensidad de la interacción y las normas informales. ${ }^{34}$ Todas estas variables constituyen una parte clave en las negociaciones aunque a menudo son ignoradas. Lewis muestra como todos estos factores favorecen el comportamiento cooperativo en las negociaciones y la socialización de sus miembros. ${ }^{35} \mathrm{Si}$ bien parece indudable la importancia de estos factores en las negociaciones, es difícil a nivel empírico mostrar el grado de influencia que tienen.

Asimismo, surge la pregunta: ¿Qué ocurre con los nuevos Estados miembros que han ido incorporándose a la UE en los últimos años? Estos países carecen del tiempo necesario de exposición al proceso de toma de decisiones de la UE y, por tanto, de la experiencia clave para la socialización de sus negociadores. ${ }^{36}$ En ausencia de esta supuesta socialización, los nuevos países supuestamente optarían por estrategias más conflictivas. ${ }^{37}$

Hay algunos estudios que se han mostrado críticos con la idea de que la socialización explique el comportamiento de los negociadores en la UE. Por ejemplo, Beyers, a partir de un estudio cuantitativo sobre 170 grupos de trabajo en el Consejo, muestra que los factores domésticos son los que siguen dominando estas negociaciones en contraste con el argumento del método comunitario y de la socialización. ${ }^{38}$ En un estudio sobre 439 propuestas legislativas, Häge tampoco aprecia un efecto sobre la socialización entre los grupos de trabajo del Consejo mientras que sí destaca la influencia que tienen las reglas del voto y el papel del Parlamento Europeo. Respecto a las grandes cumbres, Moravcsik destaca el carácter marcadamente intergubernamental de estas negociaciones..$^{39}$ Los negociadores son los representantes de los Estados que reciben órdenes de sus respectivos ejecutivos y

${ }^{33}$ Lewis «The Impact of Institutional Environments on Negotiation Styles in EU Decision Making».

${ }^{34}$ Lewis, Jeffrey, «How Institutional Environments Facilitate Cooperative Negotiation Styles in EU Decision Making» Journal of European Public Policy 17, n. 5 (2010a): 64864 y Lewis «The Impact of Institutional Environments on Negotiation Styles in EU Decision Making», 40.

35 Lewis, Jeffrey «The Impact of Institutional Environments on Negotiation Styles in EU Decision Making» Journal of European Public Policy 17, n. ${ }^{\circ}$ 5, (2010b): 650-66.

${ }^{36}$ Lewis «Is the "Hard Bargaining" Image of the Council Misleading? The Committee of Permanent Representatives and the Local Elections Directive».

${ }^{37}$ Dür y Mateo, «Bargaining Power and Negotiation Tactics: The Negotiations on the EU's Financial Perspective, 2007-2013», 567.

38 Beyers, Jan «Multiple Embeddedness and Socialization in Europe: The Case of Council Officials» International Organization, 59 (2005): 899-936.

${ }^{39}$ Moravcsik, Andrew The Choice for Europe (New York: Cornell University Press: 1998). 
defienden estratégicamente sus preferencias nacionales. Ni el contexto institucional en que se negocia, ni el hecho de formar parte de la UE tienen influencia en los resultados. Para Moravcsik las negociaciones en la UE son simplemente negociaciones intergubernamentales cuyo resultado refleja el mínimo común denominador.

La búsqueda del consenso es también un elemento particular en las negociaciones entre los grupos de trabajo del Consejo. ${ }^{40}$ A pesar de que formalmente muchas de las decisiones entre Ministros y a niveles inferiores deben aprobarse por mayoría cualificada, es ampliamente sabido que a menudo buscan el acuerdo de todos los miembros. Esto es así hasta el punto de que alrededor del $80 \%$ de la legislación se aprueba por unanimidad. ${ }^{41}$ Esta implícita «cultura del consenso» es una garantía para los Estados de que ninguna decisión será aprobada sin su consentimiento. ${ }^{42}$

\section{Preferencias y la política doméstica}

Respecto a las preferencias, Dür y Mateo destacan dos canales a partir de los cuales éstas pueden impactar en las estrategias de los países. ${ }^{43}$ Por un lado, si un país no está muy interesado en alcanzar un acuerdo, será más partidario de usar estrategias conflictivas que si tiene interés en que se tome una decisión al respecto. Por otro lado, los países que están en una posición en que pueden perder, van a recurrir más a menudo a estrategias conflictivas. Lo interesante de este estudio es que ofrece un modo dinámico de entender por qué en una ocasión unos países optan por un tipo de estrategias y no por otras. En una dirección parecida, McKibben muestra que los Estados que están más restringidos por sus respectivas políticas domésticas, sometidos al escrutinio de su electorado o sujetos a un futuro proceso de ratificación están más predispuestos a usar estrategias menos cooperativas. ${ }^{44} \mathrm{La}$

${ }^{40}$ Elgström y Jönsson, «Negotiating in the European Union: bargaining or problem solving», 698 .

${ }^{41}$ Hayes-Renshaw y Wallace, The Council... Mikko Mattila «Voting and Coalition in the Council after the Enlargement» en Unveiling the Council of the EU. Games Governments Play in Brussels ed. por Daniel Naurin y Helen Wallace (Basingstoke: Palgrave Macmillan, 2008): 23-35. Mikko Mattila, «Roll Call Analysis of Voting in the EU Council of Ministers after the 2004 Enlargement» European Journal of Political Research, 48, n. 6 (2009): 840-57.

42 Conceiçao-Heldt, «Integrative and distributive bargaining in the EU: what difference that it make?», 148 .

43 Dür, Andreas y Gemma Mateo, «Actor Characteristics and Negotiation Tactics in the EU».

${ }_{44}$ McKibben, Heather «The Effects of Power and Structure on State Bargaining Strategies» American Journal of Political Science 57, n. ${ }^{\circ} 2$ (2013): 420-21. 
presión doméstica ha sido considerada en algunos estudios como una importante fuente de poder. ${ }^{45}$

Mattila muestra que la distribución de las preferencias afecta a la capacidad del Consejo además de las reglas de votos. ${ }^{46}$ En particular, las sucesivas ampliaciones han supuesto que haya más diferencias entre las preferencias de los países viejos y los nuevos lo que hace más difíciles las negociaciones. ${ }^{47}$ Zimmer et al. en su estudio sobre las preferencias de los países en el Consejo, a partir de datos obtenidos en entrevistas, concluye que los nuevos Estados aumentan considerablemente la heterogeneidad de las preferencias en las negociaciones. ${ }^{48}$ En particular, aprecian que los países con menos recursos económicos, procedentes del norte y del este, son los que piden una mayor regulación del mercado y menos costes de producción. La conclusión es que las preferencias de los países dependen de si son contribuyentes netos o no. Kaeding y Selck sugieren que el aumento de países en la UE va a reemplazar la dimensión norte-sur por la de norte-sur-este. ${ }^{49}$

\section{El poder de los Estados}

El poder relativo de los Estados ha sido sin duda uno de los factores más estudiados a la hora de determinar la elección de estrategias. Es indiscutible que el poder de los Estados determina su comportamiento al menos en cierta medida. ${ }^{50}$ En particular, Dür y Mateo muestran que si todos los otros factores se mantienen constantes, el poder de los Estados es el factor determinante en la elección de estrategias. ${ }^{51}$ En su estudio muestran que los

45 Moravcsik, The Choice...; Dür y Mateo, «Bargaining Power and Negotiation Tactics: The Negotiations on the EU's Financial Perspective, 2007-2013».

${ }^{46}$ Mattila, Mikko, «Roll Call Analysis of Voting in the EU Council of Ministers after the 2004 Enlargement», 842.

47 Mattila, Mikko, «Roll Call Analysis of Voting in the EU Council of Ministers after the 2004 Enlargement», 842; Thomas König y Thomas Bräuninger, «Accession and Reform of the European Union: A Game Theoretical Analysis of Eastern Enlargement and the Constitutional Reform» European Union Politics, 5 (2004): 419-39.

48 Zimmer, Christina, Gerald Schneider y Michael Dobbins «The contested Council: Conflict dimensions of an intergovernmental EU institution» Political Studies, 53 (2005): 417.

${ }^{49}$ Kaeding, Michael y Torsten J. Selck, «Mapping out political Europe: Coalition patterns in EU decision-making» International Political Science Review, 25 (2005): 283.

${ }^{50}$ Entre ellos Elgström y Jönsson, «Negotiating in the European Union: bargaining or problem solving»; Dür y Mateo, «Bargaining Power and Negotiation Tactics: The Negotiations on the EU's Financial Perspective, 2007-2013»; McKibben «The Effects of Power and Structure on State Bargaining Strategies».

${ }^{51}$ Dür y Mateo, «Bargaining Power and Negotiation Tactics: The Negotiations on the EU's Financial Perspective, 2007-2013», 563-64. 
países más poderosos son los que optan por estrategias más conflictivas que denominan «hard bargaining tactics», mientras que los países menos poderosos por estrategias más cooperativas llamadas «soft bargaining tactics». Dür y Mateo analizan las estrategias de los Estados en las negociaciones sobre las Perspectivas Financieras de los años 2007-13 y muestran que «los países grandes, con una buena alternativa para negociar un acuerdo y los gobiernos que tienen constricciones nacionales, son más favorables a utilizar estrategias más conflictivas o «hard bargaining» que los Estados menos poderosos.» ${ }^{52}$ La razón está en que el uso de estas estrategias menos cooperativas sólo parece creíble en los países más poderosos.$^{53}$ En cambio, según su argumento se espera que los países menos poderosos utilicen estrategias más cooperativas.

Esta tesis ha sido confirmada por McKibben quien destaca que los Estados más poderosos son los que más recurren a estrategias conflictivas mientras que los Estados menos poderosos son los que utilizan más estrategias cooperativas.$^{54}$ Más recientemente, Zahariadis de nuevo confirma el argumento de Dür y Mateo de que el poder de los Estados es el factor determinante en la elección de estrategias. Sin embargo, señala que falta teorizar sobre los posibles cambios de estrategia y los motivos que ocasionan esos cambios. ${ }^{55}$ Sin duda este podría ser un campo de estudio en el que seguir avanzando.

\section{Instituciones}

El contexto institucional en el que se desarrollan las negociaciones también ha sido considerado fundamental en la literatura. Tanto el Consejo como el Consejo Europeo están rodeados de una serie de reglas formales e informales que guían su funcionamiento de ahí que muchos estudiosos hayan intentado ver qué impacto tienen estas reglas. ${ }^{56}$ En general, cuanto más

52 Dür y Mateo, «Bargaining Power and Negotiation Tactics: The Negotiations on the EU's Financial Perspective, 2007-2013», 557

53 Dür y Mateo, «Bargaining Power and Negotiation Tactics: The Negotiations on the EU's Financial Perspective, 2007-2013», 559.

${ }^{54}$ McKibben «The Effects of Power and Structure on State Bargaining Strategies», 42324.

55 Nicolaos Zahariadis «Bargaining power and negotiation strategy: examining the Greek bailouts, 2010-2015» Journal of European Public Policy (2016) y Dür y Mateo, «Bargaining Power and Negotiation Tactics: The Negotiations on the EU's Financial Perspective, 20072013».

56 Mateo, Hacia... 
institucionalizado es el contexto de la negociación más se espera que se utilicen estrategias integrativas o cooperativas y viceversa. ${ }^{57}$

Sobre este particular, McKibben ha llevado a cabo una importante investigación empírica a través de la cual muestra que la elección de estrategias de los Estados miembros viene determinada por la estructura en que se llevan a cabo las negociaciones ${ }^{58}$ McKibben llega a la conclusión de que ni el poder que confiere el voto ni las capacidades materiales de los Estados son determinantes en la elección de sus estrategias. ${ }^{59}$ Para analizar la variación en la utilización de estas estrategias, McKibben estudia 23 interacciones distintas sobre la política medio ambiental, la política comercial, la distribución del presupuesto comunitario, las misiones de política exterior civil y la armonización del derecho criminal entre los años 2004 y $2010 .{ }^{60} \mathrm{Su}$ conclusión es que las reglas contextuales son el factor más influyente en la dinámica de la negociación entre los países a nivel del Consejo. ${ }^{61}$

Una característica singular de las negociaciones en el Consejo y, en particular, en el Consejo Europeo que puede ser clave a la hora de explicar el comportamiento de los Estados es la necesaria unanimidad. ${ }^{62} \mathrm{Ya}$ a finales de los años ochenta, Scharpf hablaba de la «trampa de la decisión conjunta» y el riesgo de que la unanimidad provocase cierto inmovilismo en determinadas políticas. ${ }^{63} \mathrm{El}$ temor es que el interés nacional prevalezca en detrimento del avance de la integración europea. Slapin, por ejemplo, en un estudio enfocado en las negociaciones del Tratado de Amsterdam (1997) muestra que el consenso es insuficiente para alcanzar las reformas institucionales necesarias. ${ }^{64}$ Esta visión no es compartida por otros autores que consideran que la unanimidad sí puede facilitar las negociaciones. Conceiçao-Heldt hace hincapié, en su estudio sobre las negociaciones sobre pesca, que la unanimidad no siempre desemboca en el mantenimiento del

57 Dür, Andreas y Gemma Mateo, «Actor Characteristics and Negotiation Tactics in the EU»; McKibben «The Effects of Power and Structure on State Bargaining Strategies»

${ }_{58}$ McKibben, Heather, «Issue Characteristics, Issue Linkage, and States' Choice of Bargaining Strategies in the European Union» Journal of European Public Policy, 17, n. ${ }^{\circ} 5$ (2010): 694-707.

59 McKibben «The Effects of Power and Structure on State Bargaining Strategies», 423.

${ }^{60}$ McKibben, State..., 118.

${ }^{61}$ McKibben, State..., 120.

${ }^{62}$ Elgström y Jönsson, «Negotiating in the European Union: bargaining or problem solving», 691 .

${ }^{63}$ Fritz W. Scharpf, «The Joint-Decision Trap: Lessons from German Federalism and European Integration» Public Administration 66, n. ${ }^{\circ} 3$ (1988): 277-304.

${ }^{64}$ Slapin «Bargaining Power at Europe's Intergovernmental Conferences: Testing Institutional and Intergovernmental Theories», 155. 
status quo. ${ }^{65}$ Muestra que, en las negociaciones más integrativas, la formación de coaliciones y la vinculación entre distintos temas facilitan los compromisos; mientras que en las negociaciones más distributivas la Comisión puede ser de gran ayuda en la búsqueda de compromisos.

De hecho, McKibben observa que se producen diferencias en la utilización de estrategias en función del tipo de voto. ${ }^{66}$ En particular, concluye que los Estados utilizan más estrategias cooperativas en los temas que se aprueban por mayoría cualificada, mientras que, por el contrario, sus estrategias son más conflictivas cuando deciden por unanimidad. No obstante, sobre este punto habría que tomar en consideración el hecho de que las decisiones que se aprueban por mayoría cualificada son siempre necesariamente las menos conflictivas. Otros autores han destacado que la unanimidad tiene la ventaja de que permite mejor legitimar las decisiones en cada país ante la opinión pública e implementarlas a nivel nacional. ${ }^{67}$ Para los nuevos Estados ha supuesto una garantía de que no se aprueban decisiones contrarias a sus intereses y para los Estados viejos una garantía de que se defienden sus intereses nacionales pero también comunitarios. Según Mattila, los nuevos Estados de la UE parecen fácilmente haberse adaptado o interiorizado esta cultura del consenso. ${ }^{68}$

\section{Cultura}

La cultura puede explicar por qué unos países son más favorables a escoger unas estrategias que otras ${ }^{69}$ Por cultura entendemos una serie de pautas de comportamiento, creencias, normas y valores que implícitamente comparten un grupo de individuos. La idea detrás de estos estudios es que las normas culturales aprendidas prescriben el comportamiento que es apropiado seguir en una determinada situación..$^{70}$ Por ejemplo, en una cultura

65 Eugenia Conceiçao-Heldt, «Integrative and distributive bargaining in the EU: what difference that it make?» Negotiation Journal, 22, n. 2 (2006): 162.

${ }^{66}$ McKibben «The Effects of Power and Structure on State Bargaining Strategies»

${ }^{67}$ Mattila, Mikko, «Roll Call Analysis of Voting in the EU Council of Ministers after the 2004 Enlargement», 842.

68 Mattila, Mikko, «Roll Call Analysis of Voting in the EU Council of Ministers after the 2004 Enlargement», 844.

69 Dür, Andreas y Gemma Mateo, «Actor Characteristics and Negotiation Tactics in the EU».

70 Véase entre ellos: Adair, Wendi, Brett, J., Lempereur, A., Okumura, T., Shikhirev, P., Tinsley, C. and Lytle, A. «Culture and negotiation strategy», Negotiation Journal, 20 n. ${ }^{\circ} 1$ (2004): 87-111; Faure, G. O., «Cultural aspects of international negotiation» en International Negotiation: Actors, Structure, Values ed. por Peter Berton, Hiroshi Kimurra y William Zartman (London: Macmillan, 1999) 11-31; Gelfand, Michele y Naomi Dyer, «A cul- 
puede ser inapropiado el uso de determinadas tácticas conflictivas mientras que en otras culturas puede ser algo completamente habitual. Las normas culturales también ayudan a interpretar y, hasta incluso juzgar, de un modo u otro el comportamiento de los negociadores. Por ejemplo, en culturas donde el uso de tácticas más conflictivas no es corriente, va a ser muy mal visto su utilización.

Dür y Mateo derivan cuatro interesantes hipótesis basadas en la literatura. ${ }^{71}$ Según la primera hipótesis, los negociadores de países con culturas más individualistas optarán por estrategias conflictivas mientras que los negociadores de países con culturas más colectivistas optarán por estrategias menos conflictivas. Por una cultura individualista entendemos aquella en que los individuos están preocupados por su propio éxito, están expuestos a situaciones competitivas y están más habituados a comportamientos más conflictivos; mientras que una cultura colectivista es aquella en que los individuos se sienten parte de un grupo y tienen un sentimiento de interdependencia que les hace obrar de un modo más cooperativo.

La segunda hipótesis establece que los países con una democracia mayoritaria son más favorables a estrategias conflictivas en contraste con los países con una democracia consensual. Esta diferencia en la cultura política de un país puede también afectar el estilo en que negocian. Mientras que en la democracia consensual los políticos tienen que encontrar un compromiso en muchos temas lo que incentiva que estén expuestos a continuas negociaciones, en cambio los políticos en la democracia mayoritaria están más habituados a buscar simples mayorías.

La tercera hipótesis se fundamenta en el estilo diplomático de los negociadores. Algunos estudios se han ocupado de analizar las diferencias en el estilo de negociar entre países. ${ }^{72}$ Los países con una cultura diplomática enfocada en el consenso serán más partidarios de utilizar estrategias cooperativas, mientras que aquellos países que por el contrario toman una posición más de autoafirmarse recurrirán a estrategias más conflictivas. Por último, los países que se han adherido más recientemente a la UE pueden ser más

tural perspective on negotiation: progress, pitfalls, and prospects», Applied Psychology, 49, n. 1 (2000): 62-99; Metcalf, Lynn E., Allan Bird, Mark F. Peterson, Mahesh Shankarmahesh y Terri R. Lituchy, «Cultural influences in negotiations: a four country comparative analysis» International Journal of Cross-Cultural Management 7, n. ${ }^{\circ} 2$ (2007): 147-68; Salacuse, Jeswald W., «Ten ways that culture affects negotiating style: some survey results» Negotiation Journal, 14, n. 3 (1998): 221-35.

${ }^{71}$ Dür, Andreas y Gemma Mateo, «Actor Characteristics and Negotiation Tactics in the EU».

72 Cogan, Charles, French Negotiating Behavior: Dealing with La Grande Nation, Washington: US Institute of Peace Press, 2003; Smyser, W. How Germans Negotiate: Logical Goals, Practical Solutions (Washington: US Institute of Peace Press, 2002). 
favorables a recurrir a estrategias conflictivas. Este argumento se basaría en que los nuevos países no están habituados a los patrones o los modos de negociar en la UE (la «cultura del compromiso») y eso puede derivar en que actúen en un modo más defensivo. ${ }^{73}$

Sobre este tema, sería interesante avanzar en la investigación. La incorporación de nuevos Estados miembros a la UE, introduce nuevas culturas y, por consiguiente, nuevos estilos de negociar. Habría que pensar en nuevos métodos de estudio para mostrar cómo las creencias, las normas sociales, los valores, etc. de las distintas culturas pueden impactar en las estrategias que los países escogen.

\section{Estadio o nivel de las negociaciones}

Las negociaciones en el Consejo se caracterizan por que siguen una serie de niveles o estadios. Desde los más técnicos, compuestos generalmente por los consejeros de las Representaciones Permanentes de los Estados, hasta el nivel más alto entre Ministros en las distintas formaciones. Entre estos dos niveles, están las reuniones entre el grupo Mertens y el grupo Antici que son los órganos que a su vez preparan las reuniones del COREPER I y II cuyos encuentros preceden a las de los Ministros. Esta multiplicidad de reuniones y niveles tiene como objetivo simplificar el trabajo de los Ministros los cuales se reúnen entre tres y cuatro veces al año y cuya agenda incluye solamente las cuestiones más controvertidas.

En concreto, Peterson recomienda tener en cuenta los distintos niveles de análisis teniendo en cuenta los actores que dominan el proceso de toma de decisiones. ${ }^{74}$ Por ejemplo, sugiere tener en cuenta las decisiones más históricas y las que son más técnicas. En una línea bastante similar, Peters predice también mayores dificultades en los niveles más altos de la toma de decisiones que en el día a día donde las negociaciones se concentran en temas más técnicos. ${ }^{75}$ Más recientemente, Elgström y Jönsoon consideran el estadio del proceso de las negociaciones como el factor más importante que predice el comportamiento de los actores. ${ }^{76}$ Niemann coincide en que en los

73 Lewis «Is the "Hard Bargaining" Image of the Council Misleading? The Committee of Permanent Representatives and the Local Elections Directive».

${ }^{74}$ Peterson, John «Decision-making in the European Union: towards a framework for analysis» Journal of European Public Policy 2, n. ${ }^{\circ} 1$ (1995): 69-93.

75 Peters, Guy B., «Escaping the joint decision trap: repetition and sectoral politics in the European Union», West European Politics, 2, n.1 (1997): 22-36.

${ }^{76}$ Elgström y Jönsson, «Negotiating in the European Union: bargaining or problem solving», 692-93. 
estadios o las fases iniciales, es decir, durante las negociaciones más técnicas entre consejeros, la negociación es más integrativa mientras que en las fases finales es más distributiva. ${ }^{77}$ Las negociaciones en el día a día, gracias a un proceso de aprendizaje, se basan en simples ejercicios de resolución de problemas. ${ }^{78}$ Los temas controvertidos pasan a discutirse a nivel de Ministros y de los Jefes de Estado o de Gobierno. ${ }^{79}$ De ahí que pueda esperarse que en las fases finales las estrategias de los países sean más conflictivas.

\section{Temas}

Las estrategias que los actores utilizan pueden variar según el tema sobre el que se negocia. Elgström y Jönsson explican la conducta de los actores en función del nivel de politización del tema en que se discute.$^{80} \mathrm{La}$ politización se refiere a que un tema despierta un alto nivel de interés político y/o público lo que genera más conflicto. Asimismo, los autores relacionan el nivel de politización con si pertenece a la «high politics» o a la «low politics». A menor politización de un tema, más se espera un comportamiento cooperativo. Los temas menos politizados son generalmente los más técnicos, o de «low politics». ${ }^{81}$ McKibben deriva una hipótesis según la cual los Estados recurren a estrategias conflictivas en los temas más politizados, mientras que en los temas menos politizados los Estados escogerán estrategias más cooperativas. ${ }^{82}$ Es interesante que McKibben no observa que se produzca relación alguna entre la publicidad de un tema y las estrategias que escogen los Estados. ${ }^{83}$

Respecto al tipo de política que se discute, basándonos en la clasificación de Lowi hay cuatro tipos de políticas: la constitutiva que corresponde

77 Niemann «Between Communicative Action and Strategic Action: The Article 113 Committee and the Negotiations on the WTO Basic Telecommunications Services Agreement».

${ }^{78}$ Elgström y Jönsson, «Negotiating in the European Union: bargaining or problem solving»; Lewis «Is the "Hard Bargaining" Image of the Council Misleading? The Committee of Permanent Representatives and the Local Elections Directive».

${ }^{79}$ Lewis, «How Institutional Environments Facilitate Cooperative Negotiation Styles in EU Decision Making. »

${ }^{80}$ Elgström y Jönsson, «Negotiating in the European Union: bargaining or problem solving», 691-92.

${ }^{81}$ Elgström y Jönsson, «Negotiating in the European Union: bargaining or problem solving».

${ }^{82}$ McKibben, «Issue Characteristics, Issue Linkage, and States' Choice of Bargaining Strategies in the European Union»; McKibben «The Effects of Power and Structure on State Bargaining Strategies».

${ }^{83}$ McKibben «The Effects of Power and Structure on State Bargaining Strategies». 
a los niveles más alto de las negociaciones como las conferencias intergubernamentales que revisan los Tratados comunitarios; la redistributiva en que los recursos financieros se transfieren de unos actores a otros; la distributiva en que los fondos comunitarios son distribuidos en sectores; la regulativa en que se aprueban las nuevas regulaciones. ${ }^{84} \mathrm{El}$ comportamiento de los actores se diferencia en función del tipo de política que se discute. Según Elgström y Jönsson, las negociaciones sobre políticas constitutivas tienden a ser conflictivas ya que las decisiones que se aprueban tienen un fuerte impacto en los Estados. ${ }^{85}$ Las redistributivas, aunque tienen un componente de conflicto, se caracterizan porque a menudo forman parte de un «package-deal» en que todos los actores salen ganando. Las negociaciones sobre políticas distributivas suelen producirse entre expertos en un modo más cooperativo, es decir, basándose en la búsqueda de la resolución de problemas. Por último, las negociaciones sobre políticas regulativas son a menudo legales lo que implica que la Comisión Europea es un actor fundamental ya que presenta el borrador. Estas negociaciones a menudo pueden ser duras ya que suponen una pérdida de autonomía para los Estados.

\section{Euroescepticismo}

Una pregunta interesante es: ¿qué pasa con los países más euroescépticos y los más pro-europeos? ¿Existen diferencias en cuanto a las estrategias que escogen? Según algunos estudios, el electorado y las posibles dificultades de un país en el proceso de ratificación conceden poder a los negociadores. Dür y Mateo examinan la hipótesis de que los gobiernos de los países con poblaciones más euroescépticas confían más en estrategias conflictivas en las negociaciones en la UE que los gobiernos con poblaciones más pro-europeas ${ }^{86}$ Sin embargo, sería interesante seguir estudiando el impacto de la opinión pública en el comportamiento de los países ahora en particular con la reciente crisis económica y otros problemas como la crisis de refugiados que han aumentado el porcentaje de eurocepticismo en algunos países.

En suma, todos estos factores pueden explicar al menos en parte por qué unos países escogen unas estrategias y no otras. Sin embargo, lo que se

${ }^{84}$ Lowi, Theodore «Four systems of policy, politics and choice» Public Administration Review, 32, n. 4 (1972): 298-310.

85 Elgström y Jönsson, «Negotiating in the European Union: bargaining or problem solving».

${ }^{86}$ Dür y Mateo, «Bargaining Power and Negotiation Tactics: The Negotiations on the EU's Financial Perspective, 2007-2013», 566. 
desprende de este estudio es que no se puede a priori excluir ningún factor. Si bien todo apunta a que el poder de los Estados es clave, tanto las reglas de voto, el contexto institucional en que se negocia, el tipo del tema que se discute, las preferencias de los países y la cultura, etc. pueden influir el comportamiento de los negociadores. En particular, si tenemos en cuenta que cada negociación es diferente y que, por tanto, un factor que puede ser determinante en una negociación quizá no lo sea tanto en otra. Por tanto, no basta identificar los factores que pueden ser importantes sino también cuándo unos son más importantes que otros y por qué.

La lista de factores que hemos estudiado no es exhaustiva. Otros aspectos como la corriente política de los partidos políticos que representan a los países puede tener impacto en la elección de estrategias. El factor temporal es también muy interesante, los Estados probablemente han cambiado y cambian sus estrategias a lo largo del tiempo. Sería importante seguir trabajando sobre todos estos temas.

\section{Poder}

¿Por qué en algunas ocasiones unos Estados ganan y otras veces pierden en las negociaciones en la UE? ¿Qué determina el poder de los Estados? La literatura es aún escasa en este campo.$^{87} \mathrm{La}$ dificultad que conlleva estudiar y medir el poder así como el secretismo que rodea este tipo de negociaciones son probablemente las causas principales. No hay consenso en cuanto a la definición de poder. Algunos lo ven como una característica que posee un actor, mientras que otros lo ven como una causa. Los primeros se enfocan en estudiar los recursos de los actores y los segundos las preferencias de los actores y los resultados de las negociaciones. Respecto a medir el poder, la dificultad reside en que tendríamos que saber cuál sería el resultado sin que ningún actor ejerciese ningún tipo de poder. Asimismo, es difícil distinguir entre poder y suerte. ${ }^{88}$ Un acuerdo puede ser beneficioso para más de un actor envuelto en la negociación, lo que hace difícil saber cuál de ellos utilizo su poder y, en definitiva, influyó el resultado. Todo esto hace que no sea fácil medir el poder en una negociación. En esta sección, analizamos la literatura que estudia los factores que determinan el poder entre los países en el Consejo y en el Consejo Europeo.

87 Bailer, «What Factors Determine Bargaining Power in the EU?», 743.

88 Warntjen, «The Council Presidency Power Broker or Burden? An Empirical Analysis», 320 . 


\section{Poder del voto}

El Consejo establece dos posibles sistemas de voto: la unanimidad que requiere el voto a favor de todos los Estados o, al menos, su abstención y el voto por mayoría cualificada que requiere a partir del 1 de noviembre de 2014 el voto favorable del 55\% de los países (este porcentaje supone que debe aprobarse por un mínimo de 16 de los 28 Estados que componen la UE) y además los votos a favor de los países tienen que sumar como mínimo el $65 \%$ de la población de la UE. Esta doble mayoría garantiza que las decisiones se aprueben por un mínimo de países pero también por un mínimo de la población total de la UE. El 80\% de toda la legislación de la UE se adopta teóricamente siguiendo este procedimiento, aunque como hemos dicho anteriormente, sigue imperando la búsqueda del consenso.

Este procedimiento de voto por mayoría cualificada puede utilizarse para medir el poder entre los países en la UE. De ahí que existan distintos estudiosos que se ocupan de calcular índices de poder basándose en la ponderación de los votos en el Consejo lo que les permite hacer predicciones sobre el poder de los Estados ${ }^{89}$ Esta literatura ha sido criticada entre otros por Garrett y Tsebelis..$^{0}$ En particular, se critica el hecho de que estos estudios no tienen en cuenta las preferencias de los Estados y, algo más fundamental, no pueden medir el poder cuando se decide por unanimidad algo que es habitual.

Existe otro grupo de estudios que también estudian la distribución de poder que establece la UE con el voto por mayoría cualificada, pero sin calcular índices del poder. ${ }^{91}$ Estos estudios se han enfocado en el Consejo y en los comités o grupos de trabajo ya que sólo en estos niveles está previsto el voto por mayoría cualificada. Se trata de estudios muy interesantes porque arrojan información sobre el comportamiento de voto en distintos temas o en distintas fases de la negociación. Hayes-Renshaw et al., a partir de un estudio que abarca los años 1994-2004 muestran que gran parte de las deci-

89 Entre otros véase: Kaisa Herne y Hannu Nurmi, «The Distribution of A Priori Voting Power in the EC Council of Ministers and the European Parliament» Escandivanian Political Studies, 16, n. ${ }^{\circ} 3$ (1993): 269-83; Hosli, «Coalition and Power: Effects of Qualify Majority Voting in the Council of the European Union»; König, Thomas y Thomas Bräuninger, «The Inclusiveness of European Decision Rules» Journal of Theoretical Politics, 10 (1998): 12542; Tapio Raunio y Matti Wiberg «Winners and Losers in the Council: Voting Power Consequences of EU enlargement» Journal of Common Market Studies, 36 (1998): 549-62; Hosli, Madeleine, «Power Connected Coalitions, and Efficiency: Challenges to the Council of the European Union» International Political Science Review, 20, n. 4 (1999): 371-91.

${ }_{90}$ Garrett, Geoffrey y George Tsebelis «An Institutional Critique of Intergovernmentalism» International Organization, 50, n. ${ }^{\circ} 2$ (1996): 269-99.

91 Hayes-Renshaw y Wallace, The Council... 
siones se toman por consenso y en los niveles inferiores del Consejo. ${ }^{92}$ Una apreciación interesante es que existen diferencias en el voto según los distintos temas. En agricultura, pesca y mercado interior los Estados acostumbran a recurrir al voto por mayoría cualificada mientras que en otros ámbitos la búsqueda del consenso sigue siendo una práctica común.

Más recientemente, Mattila también ha llegado a una serie de conclusiones interesantes sobre el comportamiento de los países cuando votan por mayoría cualificada ${ }^{93}$ En su análisis, los países con gobiernos más de derechas son los que más frecuentemente votan en contra en las decisiones en el Consejo, así como los países con una población más euroescéptica y los países más grandes. ${ }^{94} \mathrm{El}$ país que tiene la Presidencia no suele votar en contra. Sería útil profundizar en este campo y estudiar el comportamiento de los países que tienen la Presidencia cuando votan en el Consejo.

Este modo de medir el poder permite observar de modo objetivo la distribución, los cambios o las variaciones que se han ido produciendo a lo largo de las sucesivas ampliaciones. Sin embargo, tiene la restricción de que sólo permite analizar aquellas políticas en que se toman las decisiones por mayoría cualificada en el Consejo, políticas que necesariamente van a ser menos controvertidas que aquéllas que se deciden por consenso. Por supuesto, estos estudios no examinan las negociaciones en el Consejo Europeo.

\section{Poder institucional}

El poder institucional es otro de los factores que explican el poder en numerosos estudios. En cuanto a las grandes negociaciones que modifican los Tratados comunitarios, es decir, las conferencias intergubernamentales, ${ }^{95}$ Slapin resalta que el hecho de que todos los Estados tengan la capacidad de vetar una decisión es lo que determina el poder. ${ }^{96}$ Por ejemplo, en su estudio sobre las reformas institucionales aprobadas en el Tratado de Amster-

${ }^{92}$ Hayes-Renshaw, Fiona, Wim Van Aken y Helen Wallace, «When and Why the EU Council of Ministers Votes Explicitly» Journal of Common Market Studies, 44, n. ${ }^{\circ} 1$ (2006): 183.

93 Mattila, Mikko, «Contested decisions: Empirical analysis of voting in the European Union Council of Ministers» European Journal of Political Research 43, n. ${ }^{\circ} 1$ (2004): 29-50.

94 Mattila, Mikko, «Contested decisions: Empirical analysis of voting in the European Union Council of Ministers», 47.

95 Véase sobre éstas: Mateo, Hacia...

96 Slapin, Jonathan B., «Bargaining Power at Europe's Intergovernmental Conferences: Testing Institutional and Intergovernmental Theories» International Organization, 62 (2008): 133. 
dam (1997), muestra que el poder que confiere el consenso fue determinante para que los Estados pequeños mantuviesen el status quo, su principal interés en estas negociaciones. ${ }^{97}$

Muchos trabajos muestran que determinar la agenda y guiar las negociaciones confiere poder al país que ocupa la Presidencia y a las instituciones que le asisten..$^{98} \mathrm{La}$ Presidencia actúa como mediadora en las negociaciones, la Secretaria General asiste a la Presidencia y, por último, la Comisión Europea que, aunque no tiene poder de voto, está presente en todas las negociaciones. ${ }^{99}$ La Presidencia desarrolla labores administrativas y de organización, diseña las prioridades políticas de su mandato, media entre los distintos Estados en la búsqueda de compromisos y representa al Consejo en distintas instituciones, órganos y comités. ${ }^{100}$ Algunas de estas funciones han sido ligeramente modificadas en el Tratado de Lisboa (2009) de ahí que sería interesante estudiar el nuevo rol de la Presidencia. ${ }^{101}$

Existe cierta división en la literatura sobre si el país que ocupa la Presidencia tiene más poder o si, por el contrario, limita su poder a la hora de defender sus intereses nacionales. ${ }^{102}$ Tallberg desarrolla una teoría sobre el li-

${ }^{97}$ Slapin, «Bargaining Power at Europe's Intergovernmental Conferences: Testing Institutional and Intergovernmental Theories», 155.

98 Entre algunos de estos estudios están: Dür, Andreas y Gemma Mateo, «The Irish EU Presidency and the Constitutional Treaty: Neutrality, Skills, and Effective Mediation», Irish Political Studies 23, n. ${ }^{\circ} 1$ (2008): 59-76; Slapin, «Bargaining Power at Europe's Intergovernmental Conferences: Testing Institutional and Intergovernmental Theories»; Tallberg, Bargaining...; Warntjen «The Council Presidency Power Broker or Burden? An Empirical Analysis».

99 Sobre la Presidencia véase entre otros: Schalk, Jelmer, René Torenvlied, Jeroen Weesie, y Frans Stokman, «The Power of the Presidency in EU Council Decision-making» European Union Politics, 8 (2007): 229-50; Tallberg, Jonas, «The Agenda-Shaping Powers of the EU Council Presidency» Journal of European Public Policy, 10 n. ${ }^{\circ} 1$ (2003): 1-19; Warntjen, Andreas, «The Council Presidency Power Broker or Burden? An Empirical Analysis» European Union Politics 9, n. 3 (2008): 315-38.

100 Robert Thomson, «The Council Presidency in the European Union: responsibility with power» Journal of Common Market Studies 46, n. 3 (2008): 593-617.

101 Sobre estos cambios véase: Lewis, «The Council of the European Union and the European Council».

${ }^{102}$ Entre otros: Elgström, Ole, «The honest broker? The Council Presidency as a mediator» en European Union Council Presidencies-A Comparative Perspective ed. por Ole Elgström (London: Routledge: 2003); Kollman, Ken «The Rotating Presidency of the European Council as a Search for Good Policies» European Union Politics, 4, n.1 (2003): 5174; Tallberg, «The Agenda-Shaping Powers of the EU Council Presidency»; Tallberg «The Power of the Presidency: Brokerage, Efficiency and Distribution in EU Negotiations»; Tallberg, The power...; Thomson, «The Council Presidency in the European Union: responsibility with power». Dür y Mateo, «The Irish EU Presidency and the Constitutional Treaty: Neutrality, Skills, and Effective Mediation», 
derazgo formal de la Presidencia. ${ }^{103}$ Basándose en el institucionalismo de la acción racional muestra cómo ciertas reglas, normas y procedimientos dan a la Presidencia mayor acceso tanto a la información como a los procedimientos de la negociación que al resto de Estados, lo que le facilita alcanzar acuerdos pero también conseguir sus objetivos nacionales. ${ }^{104}$ Como ejemplo, Tallberg muestra la influencia que tuvo la Presidencia alemana en los resultados de la Agenda 2000.105

Schalk et al. también estudian el poder de la Presidencia y, en concreto, en que estadio de las negociaciones es más influyente. ${ }^{106}$ Su conclusión a partir de una importante base de datos es que la Presidencia tiene mayor capacidad de influencia en el estadio final del proceso de toma de decisiones independientemente del tamaño del país y de su poder económico. Warntjen llega a una conclusión similar. ${ }^{107}$ En particular, la información privilegiada de que dispone y la presión del tiempo al final del proceso legislativo son los dos factores claves. ${ }^{108}$

Thomson et al., a partir de una impresionante base de datos sobre 70 propuestas legislativas entre 1999 y $2000,{ }^{109}$ realizan un estudio en que derivan una serie de condiciones bajo las cuales la Presidencia tiene más poder. La fase en que se aprueba una ley es el momento en que la Presidencia tiene mayor capacidad de influencia. Esta conclusión confirma la tesis defendida por Schalk et al. y Warntjen quienes mantienen que en las fases finales del proceso legislativo la Presidencia ejerce mayor influencia en su propio interés. ${ }^{110}$ Como Tallberg, Thomson sugiere que el poder de influencia de la Presidencia está también marcado por el voto por mayoría cualificada y por la posición privilegiada del Estado que ocupa la Pre-

103 Tallberg Leadership...

104 Véase: Shepsle, Kenneth A. «Studying institutions some lessons from the rational choice approach» Journal of theoretical politics 1 n. ${ }^{\circ} 2$ (1989): 131-47; Aspinwall, Mark D. y Gerald Schneider, «Same menu, seperate tables: The institutionalist turn in political science and the study of European integration» European Journal of Political Research 38 n. ${ }^{\circ} 1$ (2000): 1-36.

105 Tallberg, Jonas, «The Power of the Presidency: Brokerage, Efficiency and Distribution in EU Negotiations» Journal of Common Market Studies, 42, n. ${ }^{\circ} 5$ (2004): 999-1022.

106 Schalk et al. «The Power of the Presidency in EU Council Decision-making», 245.

107 Warntjen «The Council Presidency Power Broker or Burden? An Empirical Analysis».

108 Warntjen «The Council Presidency Power Broker or Burden? An Empirical Analysis» 335 .

109 Para más información sobre esta investigación véase: Thomson, Robert, Frans N. Stockman, Christopher M. Achen y Thomas König The European Union Decides (Cambridge: Cambridge University Press, 2006).

110 Schalk et al. «The Power of the Presidency in EU Council Decision-making»y Warntjen «The Council Presidency Power Broker or Burden? An Empirical Analysis». 
sidencia. ${ }^{111}$ Cuanto más extrema es la posición de la Presidencia, más lejos se espera que estén los resultados de su posición.

Otros estudios en cambio son más escépticos en cuanto al poder de la Presidencia en las negociaciones. ${ }^{12}$ Por ejemplo, Schout se refiere a su «nebulosa función». ${ }^{113}$ Estudia tres elementos claves de la Presidencia que están en conflicto: neutralidad, liderazgo e intereses nacionales y pone de manifiesto las dificultades que ésta tiene en la defensa de sus intereses que a menudo se convierten en «taboo». ${ }^{114}$ Tanto la neutralidad como la necesidad o responsabilidad de dirigir la Presidencia con liderazgo juegan un importante rol. De ahí que la Presidencia tenga que actuar como mediadora y se sienta obligada a combinar lo que quiere con lo que debe hacer en un modo en que preserve su reputación.

Aunque como hemos visto, la literatura sobre el rol de la Presidencia y su poder en las negociaciones es muy rica, la mayoría de estos estudios son anteriores a las últimas ampliaciones de la UE y a los cambios institucionales que ha introducido el Tratado de Lisboa (2009) lo que hace conveniente nuevos estudios. Estos deberían examinar el nuevo papel de la Presidencia del Consejo así como el del nuevo Presidente del Consejo Europeo. Un tema importante, aún poco estudiado, es cómo se coordina el trabajo entre los tres Estados que conjuntamente ocupan la Presidencia. Sin duda, la incorporación de nuevos países en la UE ha añadido nuevos desafíos a la Presidencia.

\section{Poder estructural}

El «poder estructural agregado» de los Estados ha sido también objeto de estudio. Este poder estructural está determinado por factores estrictamente geográficos, demográficos, económicos, militares, estabilidad política, etc. Según esta explicación los países que poseen más poder estructural son los más exitosos o poderosos en las negociaciones internacionales. Tallberg, a partir de una serie de entrevistas a altos cargos políticos en la

111 Tallberg «The Power of the Presidency: Brokerage, Efficiency and Distribution in EU Negotiations»; Thomson, «The Council Presidency in the European Union: responsibility with power», 612 .

112 Entre otros: Dewost, Jean-Louis, «La Présidence dans le cadre institutionnel des Communautés Européennes» Revue du Marché Commu, 273 (1984): 31-34; Desmond Dinan, Ever Closer Union? An introduction to the European Community (New York: Palgrave Macmillan, 2010); Schout, Adriaan, The Presidency as jugger: managing conflicting expectations (EIPASCOPE, 2, 1998).

113 Schout, The Presidency...

114 Schout, The Presidency..., 7. 
UE, muestra la importancia del poder estructural en las negociaciones en el Consejo Europeo es indiscutible. ${ }^{115}$ No hay duda de que el tamaño económico de los Estados es una importante fuente de poder. De acuerdo con el liberalismo intergubernamental, Moravcsik asegura que son los tres Estados más poderosos económicamente - Alemania, Francia y Reino Unido- los que determinan las negociaciones en la UE. ${ }^{116}$ El resultado de las negociaciones no es más que el mínimo común denominador según los intereses de estos tres países. El resto de Estados obtienen a cambio pequeñas compensaciones. Bailer distingue dos dimensiones de poder. ${ }^{117}$ Por un lado, una dimensión exógena que responde al número de votos y al poder económico y, por otro lado, una dimensión endógena basada en su capacidad y la información que tienen. Su conclusión es que el poder exógeno sigue siendo el factor determinante en la distribución de poder en el Consejo.

Aunque como acabamos de ver esta tesis ha sido confirmada por Bailer en el Consejo y por Morvacsik y Tallberg en el Consejo Europeo, es difícil separar el peso económico de otras variables que también pueden influir en el poder de los Estados. ${ }^{118}$ Algunos autores muestran casos concretos en que países pequeños han sido claves en las negociaciones y han influido los resultados en su propio interés. Por ejemplo, Lewis asegura que, en las negociaciones de la directiva de las elecciones locales de 1994, Bélgica alcanzó sus objetivos. ${ }^{119}$ En particular, sostiene que la «racionalidad comunicativa» es también un factor importante.

\section{Características de los negociadores}

La habilidad en la negociación, la personalidad de los negociadores, la autoridad y la pericia o experiencia de los negociadores no ha recibido mucha atención en la literatura. ${ }^{120}$ Hosli destaca la personalidad y la experiencia de los negociadores como un factor importante que puede determinar el poder. ${ }^{121}$ Tallberg destaca la autoridad personal y la experiencia como as-

115 Tallberg, Bargaining..., 14.

116 Moravcsik, The Choice...

117 Bailer, «What Factors Determine Bargaining Power in the EU? »

118 Bailer, Stephanie, «The Dimensions of Power in the European Union» Comparative European Politics, 4, n. 4 (2006): 355-78; Moravcsik, The Choice... y Tallberg, Bargaining...

119 Lewis «Is the "Hard Bargaining" Image of the Council Misleading? The Committee of Permanent Representatives and the Local Elections Directive», 499.

120 Tallberg, Bargaining...

121 Hosli, Madeleine, «Coalition and Power: Effects of Qualify Majority Voting in the Council of the European Union» Journal of Common Market Studies, 34 (1996), 256. 
pectos de gran importancia en los Consejos Europeos. ${ }^{122}$ La situación cambia en reuniones a nivel del Consejo donde sólo influye este factor en las reuniones a más alto nivel (las formaciones del Consejo) mientras que en reuniones a nivel de COREPER y de grupos de trabajo imperan otros criterios. ${ }^{123} \mathrm{El}$ grado de conocimiento y las redes de contactos entre los negociadores pueden ser también factores de poder. ${ }^{124}$ Sin embargo, es difícil llevar a cabo estudios sistemáticos en los que se muestra la influencia de las características personales de los negociadores, aunque es indiscutible que pueden tener un impacto importante.

\section{El BATNA de los países}

La Mejor Alternativa a un Acuerdo Negociado (BATNA) es también una fuente de poder. ${ }^{125}$ Se determina por las preferencias de un actor y la disponibilidad de opciones que tiene. En otras palabras, se refiere a la mejor alternativa que un actor tiene en un resultado negociado. Algunos países dependen más de las negociaciones que otros países. Según algunos estudios, este factor puede explicar por qué en algún tema particular un país tiene más poder que otro. ${ }^{126}$ En general, se puede decir que los países que pierden menos del bloqueo o el fracaso de una negociación tienen un mejor BATNA lo que les hace tener más poder en las negociaciones.

Dür y Mateo argumentan que en las negociaciones sobre las Perspectivas Financieras para el 2007 y 2013, los países contribuyentes netos tienen más poder en las negociaciones, es decir, mejor BATNA que los que reciben las ayudas. ${ }^{127}$ Esta situación viene determinada por el hecho de que si no se llega a un acuerdo durante las negociaciones, se tiene que aprobar el presupuesto anualmente a partir de 2006 lo que limita significativamente el presupuesto destinado a los países que reciben ayudas. Dado que existe

122 Tallberg, Bargaining...

123 Bailer, «The Dimensions of Power in the European Union».

124 Beyers, Jan y Guido Dierickx «Nationality and European negotiations: the working groups of the Council of Ministers» European journal of international relations $3 \mathrm{n} .^{\circ} 4$ (1997): 435-471; Naurin, Daniel y Rutger Lindahl «East, North, South. Coalition-Building in the Council Before and After Enlargement» en Unveiling the Council of the EU. Games Governments Play in Brussels ed. por Daniel Naurin y Helen Wallace (Basingstoke: Palgrave Macmillan, 2008).

125 Fisher, Roger y William Ury, Getting to Yes: Negotiating Agreement without Giving In. (London: Hutchinston Business, 1981).

${ }^{126}$ Dür y Mateo, «Bargaining Power and Negotiation Tactics: The Negotiations on the EU's Financial Perspective, 2007-2013».

127 Dür y Mateo, «Bargaining Power and Negotiation Tactics: The Negotiations on the EU's Financial Perspective, 2007-2013». 
muy poca literatura en este campo, sería interesante ver la relación entre poder y BATNA estudiando negociaciones repetidas a nivel de los comités del Consejo.

\section{Las coaliciones entre países}

Existe una amplia serie de estudios que analizan la formación y el efecto de las coaliciones en las negociaciones en la UE. ${ }^{128}$ Hosli destaca la dificultad de analizar las coaliciones de países en el Consejo. ${ }^{129}$ Tallberg concede un rol fundamental a las coaliciones entre grupos de países como el eje franco-alemán, el Benelux o los países nórdicos; también a redes entre partidos políticos en particular entre los dos grandes partidos; y, por último, a coaliciones entorno a temas específicos. ${ }^{130}$ Por ejemplo, Hosli afirma que actualmente las clásicas coaliciones entre Benelux y el eje franco-alemán han perdido importancia. ${ }^{131}$ En cambio, han surgido nuevas coaliciones entre los países que son contribuyentes netos y los que reciben ayuda. ${ }^{132}$ Kaeding y Selck formulan una serie de hipótesis sobre el impacto de las preferencias de los Estados en el Consejo en la formación de coaliciones. ${ }^{133} \mathrm{Si}$ bien todos estos estudios son muy interesantes valdría la pena actualizarlos para ver el efecto de la incorporación de nuevos Estados en la formación de coaliciones. Las últimas tres ampliaciones de la UE a partir del 2004 han supuesto la entrada de trece nuevos países, ${ }^{134}$ habría que preguntarse si han supuesto la creación de nuevas coaliciones en el Consejo. Y si es así, ¿qué poder tienen? ¿Y qué efecto tienen en las clásicas coaliciones como la de los países del sur, del Benelux o los países nórdicos?

En suma, la amplitud de estudios sobre la distribución del poder en el Consejo y en el Consejo Europeo pone de manifiesto que no se puede ex-

128 Por ejemplo: Hosli, «Coalition and Power: Effects of Qualify Majority Voting in the Council of the European Union»; Mattila «Contested decisions: Empirical analysis of voting in the European Union Council of Ministers»; Tallberg Leadership...

129 Hosli, «Coalition and Power: Effects of Qualify Majority Voting in the Council of the European Union», 257.

130 Tallberg Leadership..., 7.

131 Hosli, «Coalition and Power: Effects of Qualify Majority Voting in the Council of the European Union»

132 Mattila, «Contested decisions: Empirical analysis of voting in the European Union Council of Ministers»

${ }^{133}$ Kaeding y Selck, «Mapping out political Europe: Coalition patterns in EU decisionmaking»

${ }^{134}$ En el 2004 se incorporaron: República Checa, Chipre, Eslovaquia, Eslovenia, Estonia, Hungría, Letonia, Lituania, Malta y Polonia; en 2007: Rumanía y Bulgaria; y en 2013 Croacia. 
plicar por un único factor. Por el contrario, el poder de los Estados viene determinado por una combinación de factores. Las reglas formales e informales, el contexto de las negociaciones, el estadio de las negociaciones, las características de los países y de los temas que se discuten, entre otros factores, pueden influir la distribución del poder. Asimismo, estos factores pueden tener diferente impacto en la negociación lo que hace difícil decir cuál es más importante y por qué. Lo interesante sería enfocarse en qué factor es más importante o, mejor dicho, cuándo un factor es más importante que otro ya que no se puede entender el poder como algo estático sino que éste puede cambiar a lo largo del tiempo o en función de determinadas circunstancias. En esta sección, no hemos estudiado el poder de la Secretaria General y de la Comisión Europea, a pesar de que parece indudable que pueden influir en las negociaciones. Sería interesante enfocarse en el poder de estas dos instituciones en futuros estudios.

\section{Conclusiones}

En este trabajo hemos realizado una revisión de la literatura sobre las estrategias y el poder en las negociaciones en el Consejo y en el Consejo Europeo. Como hemos visto, hay una gran amplitud de estudios que se ocupan desde distintas perspectivas teóricas y a partir de diferentes métodos empíricos de explicar estas negociaciones en el marco de la UE. La gran riqueza de esta literatura es que mezcla distintas metodologías. Aunque en el artículo hemos optado por separar los estudios de estrategias y de poder con el objetivo de dar una mayor claridad al estudio, no cabe duda que existe una importante interrelación entre ambos. Las estrategias pueden dar mayor o menor poder a los actores y, al mismo tiempo, el poder determina las estrategias que escogen los actores. Un actor que tiene mayor poder puede escoger mejor sus estrategias mientras que un actor que carece de recursos va a tener menos capacidad a la hora de escoger sus estrategias. Esta es también una de las dificultades a la que se enfrentan muchos estudios ya que no se puede estudiar poder sin identificar las estrategias de los actores y, al mismo tiempo, sus estrategias vienen determinadas por el poder de los actores.

Existen muchas líneas de investigación abiertas. Por un lado, sería interesante integrar el impacto de la opinión pública en las negociaciones en las UE. El creciente euroescepticismo junto con el mayor y más rápido acceso a la información ofrece nuevos desafíos a los negociadores. Sería interesante saber qué impacto tiene en particular en las grandes cumbres. Asimismo, no existen muchos estudios que analizan las preferencias de los partidos políticos en los distintos Estados y su impacto en los gobiernos de 
los Estados miembros que son los que negocian. Hasta ahora la mayoría de los estudios sobre preferencias se concentran únicamente en el partido en el gobierno. Además, faltan estudios actualizados en que se tienen en cuenta los 28 países que actualmente componen la UE. No hay duda que este creciente aumento ha acelerado una serie de cambios en la dinámica de las negociaciones cuyos efectos podría ser de gran interés estudiarlos. Asimismo, el rol del presidente del Consejo Europeo y cómo éste influye en las negociaciones no ha sido aún analizado, tampoco el modo en que las tres Presidencias del Consejo se coordinan y el efecto que tiene que trabajen sobre una agenda que ocupa tres semestres. Sin duda, las coaliciones entre países es un tema fundamental. Se tiende a pensar que a más miembros en la UE, mayor necesidad de crear coaliciones. No obstante, no hay evidencia empírica de que hayan aumentado el número de coaliciones y del impacto que han podido tener. En suma, aunque existe una amplia y rica literatura sobre las negociaciones en la UE, quedan muchos campos abiertos pendientes de investigación.

\section{Bibliografia}

Adair, Wendi, Brett, J., Lempereur, A., Okumura, T., Shikhirev, P., Tinsley, C. and Lytle, A. «Culture and negotiation strategy». Negotiation Journal 20, n. ${ }^{\circ} 1$ (2004): 87-111.

Aspinwall, Mark D. y Gerald Schneider. «Same menu, separate tables: The institutionalist turn in political science and the study of European integration». European Journal of Political Research 38, n. ${ }^{\circ} 1$ (2000): 1-36.

Bailer, Stephanie. «The Dimensions of Power in the European Union». Comparative European Politics 4, n. ${ }^{\circ} 4$ (2006): 355-78.

Bailer, Stephanie. «What Factors Determine Bargaining Power in the EU?». Journal of European Public Policy 17, n. ${ }^{\circ}$ 5, (2010): 743-752.

Cogan, Charles. French Negotiating Behavior: Dealing with La Grande Nation. Washington: US Institute of Peace Press, 2003.

Jan Beyers. «Multiple Embeddedness and Socialization in Europe: The Case of Council Officials». International Organization 59, n. ${ }^{\circ}$ (2005): 899-936.

Jan Beyers y Guido Dierickx. «Nationality and European negotiations: the working groups of the Council of Ministers». European Journal of International Relations 3, n. $^{\circ} 4$ (1997): 435-71.

De Conceiçao-Heldt, Eugenia. «Integrative and distributive bargaining in the EU: what difference does it make?». Negotiation Journal 22, n. ${ }^{\circ} 2$ (2006): 145-165.

Dewost, Jean-Louis. «La Présidence dans le cadre institutionnel des Communautés Européennes». Revue du Marché Commun 273 (1984): 31-4.

Dinan, Desmond, Ever closer union? An introduction to the European Community. New York: Palgrave Macmillan, 2010. 
Dür, Andreas y Gemma Mateo. «The Irish EU Presidency and the Constitutional Treaty: Neutrality, Skills, and Effective Mediation». Irish Political Studies 23, n. 1 (2008): 59-76.

Dür, Andreas y Gemma Mateo. «Actor Characteristics and Negotiation Tactics in the EU». Journal of European Public Policy 17, n. 5 (2010a): 680-93.

Dür, Andreas y Gemma Mateo. «Bargaining Power and Negotiation Tactics: The Negotiations on the EU's Financial Perspective, 2007-2013». Journal of Common Market Studies 48, n. 3 (2010b): 557-78.

Elgström, Ole y Jönsson, Christer. «Negotiation in the European Union: bargaining or problem solving». Journal of European Public Policy 7, n. 5 (2000): 684-704.

Elgstrom, Ole. «The honest broker? The Council Presidency as a mediator», en European Union Council Presidencies-A Comparative Perspective, ed. por Ole Elgström (London: Routledge, 2003), 38-54.

Elms, Deborah. «How bargaining alters outcomes: bilateral trade negotiations and bargaining strategies». International Negotiation 11, n. o 3 (2006): 399-429.

Faure, G. O. «Cultural aspects of international negotiation», en International Negotiation: Actors, Structure, Values, ed. por Peter Berton, Hiroshi Kimurra y William Zartman (London: Macmillan, 1999), 11-31.

Fisher, Roger y William Ury, Getting to Yes: Negotiating Agreement without Giving In London: Hutchinson Business, 1981.

Garrett, Geoffrey y George Tsebelis. «An Institutional Critique of Intergovernmentalism». International Organization 50, n. 2 (1996): 269-99.

Gelfand, Michele y Naomi Dyer. «A cultural perspective on negotiation: progress, pitfalls, and prospects». Applied Psychology 49, n. ${ }^{\circ} 1$ (2000): 62-99.

Hayes-Renshaw, Fiona y Helen Wallace, The Council of Ministers. New York: Palgrave Macmillan: 2006.

Héritier, Adrienne. «The accommodation of diversity in European policymaking and its outcomes: regulatory policy as a patchwork». Journal of European Public Policy 3, n. 2 (1996): 149-67.

Herne, Kaisa y Hannu Nurmi. «The distribution of A Priori Voting Power in the EC Council of Ministers and the European Parliament». Scandinavian Political Studies 16, n. ${ }^{\circ}$ 3, (1993): 269-83.

Hopmann, Terrence P. «Bargaining in Arms Control Negotiations: The Seabeds Denuclearization Treaty». International Organization 28, n. 3 (1974): 313-43.

Hopmann, Terrence P. «Two Paradigms of Negotiation: Bargaining and Problem Solving». Annals of the American Academy of Political and Social Science 542 (1995): 24-47.

Hosli, Madeleine. «Coalitions and Power: Effects of Qualify Majority Voting in the Council of the European Union». Journal of Common Market Studies 34 (1996): 255-73.

Hosli, Madeleine. «Power Connected Coalitions, and Efficiency: Challenges to the Council of the European Union». International Political Science Review 20, n. ${ }^{\circ} 4$ (1999): 371-91.

Kaeding, Michael y Torsten J. Selck. «Mapping out political Europe: Coalition patterns in EU decision-making». International Political Science Review 25 (2005): 271-90. 
Keohane, Robert O., After Hegemony: Cooperation and Discord in the World Political Economy. Princeton: Princeton University Press, 1984.

Kollman, Ken. «The Rotating Presidency of the European Council as a Search for Good Policies». European Union Politics 4, n. ${ }^{\circ} 1$ (2003): 51-74

König, Thomas y Thomas Bräuninger. «The Inclusiveness of European Decision Rules». Journal of Theoretical Politics 10 (1998): 125-42.

König, Thomas y Thomas Bräuninger. «Accession and reform of the European Union: A gametheoretical analysis of eastern enlargement and the constitutional reform». European Union Politics 5 (2004): 419-39.

Lax, David A. y James Sebenius, The Manager as Negotiator: Bargaining for Cooperation and Competitive Gain. New York: Free Press, 1986.

Lewis, Jeffrey. «Is the "Hard Bargaining" Image of the Council Misleading? The Committee of Permanent Representatives and the Local Elections Directive». Journal of Common Market Studies 36, n. . 4 (1998): 479-504.

Lewis, Jeffrey. «The Methods of Community in EU Decision-making and Administrative Rivalry in the Council's Infrastructure». Journal of European Public Policy 7, n. 2 (2000): 261-89.

Lewis, Jeffrey. «How Institutional Environments Facilitate Cooperative Negotiation Styles in EU Decision Making». Journal of European Public Policy 17, n. ${ }^{\circ} 5$ (2010a): 648-64.

Lewis, Jeffrey. «The Impact of Institutional Environments on Negotiation Styles in EU Decision Making». Journal of European Public Policy 17(5) (2010b) 650-66.

Lewis, Jeffrey, «The Council of the European Union and the European Council», en Politics in the European Union, ed. por: Michelle Cini y Nieves Perez-Solorzano Borragan, (Oxford: Oxford University Press, 2013), 142-58.

Lowi, Theodore. «Four systems of policy, politics and choice». Public Administration Review 32, n. 4 (1972): 298-310.

Mateo Gonzalez, Gemma, Hacia una Constitución Europea: Las Conferencias Intergubernamentales en la Unión Europea. Valencia, Tirant Lo Blanch: 2008.

Mattila, Mikko, «Contested decisions: Empirical analysis of voting in the European Union Council of Ministers». European Journal of Political Research 43, n. ${ }^{\circ} 1$ (2004): 29-50.

Mattila, Mikko, «Voting and Coalition in the Council after the Enlargement», en Unveiling the Council of the European Union. Games Governments Play in Brussels, ed. por: Naurin, Daniel y Helen Wallace (Basingstoke: Palgrave Macmillan, 2008), 23-35.

Mattila, Mikko, «Roll Call Analysis of Voting in the EU Council of Ministers after the 2004 Enlargement» European Journal of Political Research 48, n. 6 (2009): 840-57.

Hayes-Renshaw, Fiona y Helen Wallace, The Council of Ministers. London: Macmillan, 1997.

Hayes-Renshaw, Fiona, Wim Van Aken, y Helen Wallace, «When and Why the EU Council of Ministers Votes Explicitly», Journal of Common Market Studies 44, n. ${ }^{\circ} 1$ (2006): 161-94.

McKibben, Heather, State strategies in international bargaining: Play by the rules or change them? Cambridge: Cambridge University Press, 2015. 
McKibben, Heather, «Issue Characteristics, Issue Linkage, and States' Choice of Bargaining Strategies in the European Union», Journal of European Public Policy 17, n. $^{\circ} 5$ (2010): 694-707

McKibben, Heather 2013 «The Effects of Power and Structure on State Bargaining Strategies», American Journal of Political Science 57, n. ${ }^{\circ} 2$ (2013): 411-27.

Metcalf, Lynn E., Allan Bird, Mark F. Peterson, Mahesh Shankarmahesh y Terri R. Lituchy, «Cultural influences in negotiations: a four country comparative analysis», International Journal of Cross-Cultural Management 7, n.o 2 (2007): 147 68.

Moravcsik, Andrew, The Choice for Europe. New York: Cornell University Press, 1998.

Naurin, Daniel y Helen Wallace, Eds. Unveiling the Council of the European Union: Games governments play in Brussels. Basingstoke: Palgrave Macmillan, 2008.

Naurin, Daniel y Rutger Lindahl, «East, North, South. Coalition-Building in the Council Before and After Enlargement», en Unveiling the Council of the EU. Games Governments Play in Brussels, ed. por: Naurin, Daniel y Wallace, Helen (Basingstoke: Palgrave Macmillan, 2008).

Niemann, Arne, «Between Communicative Action and Strategic Action: The Article 113 Committee and the Negotiations on the WTO Basic Telecommunications Services Agreement», Journal of European Public Policy 11, n. 3 (2004): 379-407.

Odell, John .S., Negotiating the World Economy. New York: Cornell University Press, 2000.

Peters, B. Guy «Escaping the joint-decision trap: repetition and sectoral politics in the European Union», West European Politics 20, n. . 2 (1997): 22-36.

Peterson, John, «Decision-making in the European Union: towards a framework for analysis», Journal of European Public Policy 2, n. ${ }^{\circ} 1$ (1995): 69-93.

Raunio, Tapio y Matti Wiberg, «Winners and losers in the Council: Voting power consequences of EU enlargement» Journal of Common Market Studies 36 n. 4 (1998): 549-62.

Risse, Thomas, «Let's Argue: Communicative Action in World Politics», International Organization 54, n. 1 (2000): 1-40.

Salacuse, Jeswald W., «Ten ways that culture affects negotiating style: some survey results», Negotiation Journal 14, n. ${ }^{\circ} 3$ (1998): 221-35.

Schalk, Jelmer, René Torenvlied, Jeroen Weesie, y Frans Stokman, «The Power of the Presidency in EU Council Decision-making», European Union Politics 8 (2007): 229-50.

Scharpf, Fritz W. «The Joint-Decision Trap: Lessons from German Federalism and European Integration». Public Administration 66, n. 3 (1988): 277-304.

Schout, Adriann, The Presidency as jugger: managing conflicting expectations. EIPASCOPE, 2 (1998).

Shepsle, Kenneth A., «Studying institutions some lessons from the rational choice approach», Journal of theoretical politics 1, n. 2 (1989): 131-47.

Sherrington, Philippa, The Council of Ministers: Political Authority in the European Union. London: Pinter, 2000. 
Slapin, Jonathan B., «Bargaining Power at Europe's Intergovernmental Conferences: Testing Institutional and Intergovernmental Theories», International Organization 62 (2008): 131-62.

Smyser, W., How Germans Negotiate: Logical Goals, Practical Solutions, Washington: US Institute of Peace Press, 2002.

Tallberg, Jonas, «The Agenda-Shaping Powers of the EU Council Presidency», Journal of European Public Policy 10, n..$^{\circ}$ (2003): 1-19.

Tallberg, Jonas, «The Power of the Presidency: Brokerage, Efficiency and Distribution in EU Negotiations», Journal of Common Market Studies 42, n. ${ }^{\circ} 5$ (2004): 999-1022.

Tallberg, Jonas. Leadership and Negotiation in the European Union: The Power of the Presidency. Cambridge: Cambridge University Press, 2006.

Tallberg, Jonas, Bargaining Power in the European Council. Working Paper SIEPS 1, 2007. file:///C:/Users/duera/Documents/Gemma/TRABAJO/Tallberg_06.pdf

Thomas, Daniel, «Explaining the negotiation of EU foreign policy: Normative institutionalism and alternative approaches» International Politics 46 (2009): 339-57.

Thomson, Robert, Frans N. Stockman, Christopher M. Achen y Thomas König, The European Union Decides. Cambridge: Cambridge University Press, 2006.

Thomson, Robert, «The Council Presidency in the European Union: responsibility with power» Journal of Common Market Studies 46, n. ${ }^{\circ}$ 3, p. (2008): 593-617.

Walton, Richard E. y Robert B. McKersie. A Behavioral Theory of Labor Negotiations: An Analysis of a Social Interaction System. Ithaca: ILR Press, 1965.

Westlake, Martin y David Galloway. Eds. The Council of the European Union. London: John Harper Publishing, 2006.

Zahariadis, Nicolaos «Bargaining power and negotiation strategy: examining the Greek bailouts, 2010-2015», Journal of European Public Policy (2016).

Zimmer, Christina, Gerald Schneider y Michael Dobbins, «The contested Council: Conflict dimensions of an intergovernmental EU institution»Political Studies 53 (2005): 403-22.

Walton, Richard y Robert McKersie, A Behavioral Theory of Labor Negotiations: An Analysis of a Social Interaction System. New York: ILR Press, 1965.

Warntjen, Andreas, «The Council Presidency Power Broker or Burden? An Empirical Analysis», European Union Politics 9, n. ${ }^{\circ} 3$ (2008): 315-38.

Werts, Jan, The European Council. London: John Harper Publishing, 2008. 


\section{Derechos de autor (Copyright)}

Los derechos de autor (distribución, comunicación pública, reproducción e inclusión en bases de datos de indexación y repositorios institucionales) de esta publicación pertenecen a la editorial Universidad de Deusto. El acceso al contenido digital de cualquier número de Cuadernos Europeos de Deusto (CED) es gratuito, transcurridos 6 meses desde su publicación. Los trabajos podrán descargarse, copiar y difundir, sin fines comerciales y según lo previsto por la ley. Así mismo, los trabajos editados en CED pueden ser publicados con posterioridad en otros medios o revistas, siempre que el autor indique con claridad y en la primera nota a pie de página que el trabajo se publicó por primera vez en CED, con indicación del número, año, páginas y DOI (si procede). 